\title{
Performance of the EUSO-Balloon optics
}

\section{Catalano*, P. von Ballmoos, B. Mot, G. Roudil, C. Smith, G. Durando}

Institut de Recherche en Astrophysique et Planétologie(IRAP), France

E-mail: ccatalanodirap.omp.eu

\section{G. Durando}

Institut de Recherche en Astrophysique et Planétologie(IRAP), France

Department of Physics, University of Torino, Italy

\section{Y. Takizawa}

RIKEN, Japan

\section{N. Sakaki}

Osaka City University, Japan

\section{for the JEM-EUSO Collaboration}

EUSO-Balloon is a prototype of the JEM-EUSO detector to perform an end-to-end test of the JEM-EUSO subsystems and components to validate the entire detection chain and to measure the atmospheric and terrestrial UV background. In August 2014, the instrument was launched in collaboration with the French Space Agency CNES for its maiden flight. This article describes the optics of EUSO-Balloon, which consists of two large $\left(1 \mathrm{~m}^{2}\right)$ Fresnel lenses made from PMMA. We also present the methods used for the alignment and characterization of the optics. The alignment of the optics was achieved with the use of a laser tracker, with tests performed using a one-meter collimator and UV light sources. The point spread function and the global efficiency, representing the performance of the optical system, are presented as functions of UV wavelength and incidence angle.

The 34th International Cosmic Ray Conference,

30 July- 6 August, 2015

The Hague, The Netherlands

\footnotetext{
* Speaker.
} 


\section{Introduction}

EUSO-Balloon is a balloon pathfinder for the Extreme Universe Space Observatory on the Japanese Experiment Module (JEM-EUSO). JEM-EUSO will be secured to the International Space Station and its mission is to observe the UV fluorescence light (between $290 \mathrm{~nm}$ and 430nm) produced by UHECR air showers in the atmosphere with a very large field of view $\left( \pm 30^{\circ}\right)[1]$. The EUSO-Balloon prototype tests the principles of the JEM-EUSO experiment by performing an endto-end test of all the subsystems and components, as well as measuring the atmospheric and terrestrial UV light in real conditions[2]. For this purpose, all subsystems have been conceived based on the JEM-EUSO design. As for the space mission, the optics of EUSO-Balloon is composed of large Fresnel lenses. The two $1 \mathrm{~m} \times 1 \mathrm{~m}$ convergent lenses are made of UV transmittance grade plastic PMMA, designed to produce a focal spot size close to the size of a pixel $(2.9 \mathrm{~mm})$ of the detector with a large field of view $\left(11^{\circ}\right)$. The optical system used during the first flight in August 2014 was the two-lens optical design named "TA-EUSO", described in [3].

In order to characterise the optics, a 1-meter-diameter collimator was used with UV LEDs at different wavelengths. The optical system was aligned with the collimated beam using a laser tracker and two theodolites. A photodiode sensor with absolute calibration was implemented at the focal plane to measure the light flux. The sensor was certified in accordance with the "National Institute of Standards and Technology". As such, for simplicity, the sensor is referred to as "NIST" throughout this article. A second test campaign took place in a long corridor. Using a mercury lamp as a light source placed at long distance, the optical system was characterised at selected wavelengths between $300 \mathrm{~nm}$ and $400 \mathrm{~nm}$.

In this article, we present the characteristics of the optics measured so far: the point spread function, the efficiency and the throughput. The size of the spot is characterised by the full width at half maximum (FWHM) of the point spread function (PSF). The efficiency is computed from the focal spot and it is relevant for the study of imaged events. The throughput, another form of efficiency, is computed from the flux received from the entire detector and is relevant for the study of the background measurement.

\section{The EUSO-Balloon optics design}

The EUSO-Balloon optics design is composed of two large PMMA Fresnel lenses to resemble the JEM-EUSO optics. Each lens has a collective area, or aperture, of $0.95 \mathrm{~m}^{2}$ and a thickness of $8 \mathrm{~mm}$. They are made with grooves on only one side: the back side for the front lens and the front side for the rear lens. The optics have been designed to resemble the JEM-EUSO optics and their performances have been calculated to reproduce a background rate close to the one predicted for the space telescope JEM-EUSO. Together with the detector efficiency, the optics have to produce a background light level of roughly 1 to 1.5 photoelectrons per pixel in a $2.5 \mu$ s frame.

The front lens and the rear lens are convergent with focal lengths equal to $2586 \mathrm{~nm}$ and $600 \mathrm{~mm}$ respectively. These two characteristics are given as ideal reference values only, due to the nonstigmatic images produced by one lens alone. Figure 1 presents pictures of the lenses and a ray tracing diagram showing the positions of the two lenses and ray traces of light for different angles of incidence. The positioning of the lenses was made using a laser tracker. This device enables us 


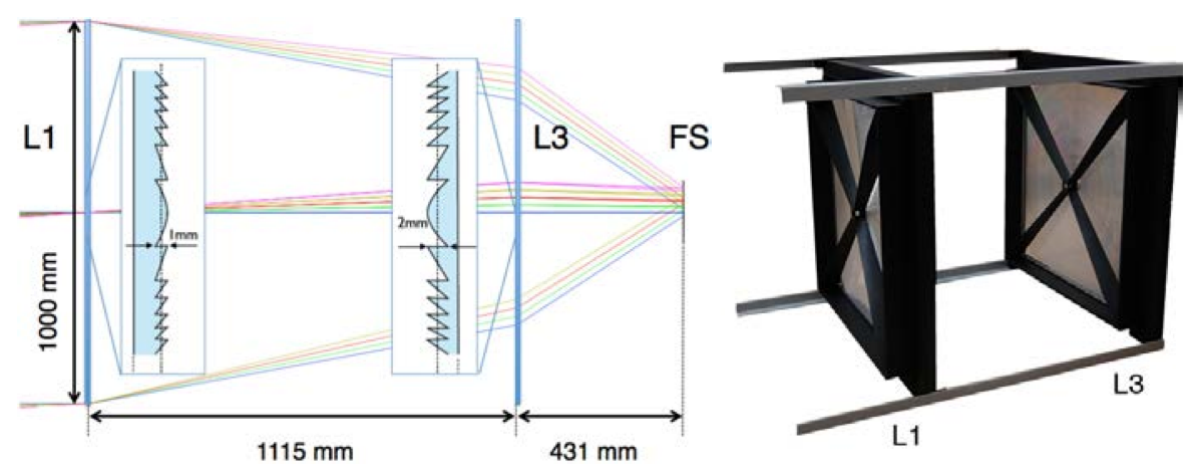

Figure 1: Left: Ray tracing diagram for the EUSO-Balloon optics with the two Fresnel lenses (front lens L1 and rear lens L3) and the position of the focal surface. The incident rays are at off-axis angles for $0^{\circ}$ (blue), $1^{\circ}$ (green), $2^{\circ}$ (red), $3^{\circ}$ (yellow) and $4^{\circ}$ (purple). The ray tracing diagram shows partial sectional views of each lens. Right: The two lenses mounted onto their fiberglass frames and spiders. Positions are adjustable.

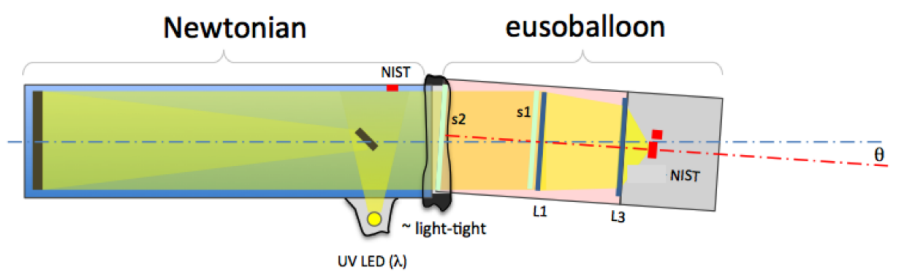

Figure 2: Diagram of the setup using the collimator to test the optics. A 1-meter-diameter collimated UV light beam was sent through the optical system. A NIST photodiode sensor was used at the focal plane to measure the focal spot.

to know the position of objects in space with a precision of a few $\mu m$. The lenses are positioned with an error smaller than $1 \mathrm{~mm}$ and are parallel within an error of less than 0.1 degree.

\section{Collimator first test campaign}

\subsection{Description of the test setup}

For this test campaign, a 1-meter-diameter collimator was used with a 390nm LED in order to create a 390nm collimated light beam. Sent through the optical system, the collimated light beam acts as a point source at infinity. The NIST photodiode was used to measure the light at the focal plane of the telescope as well as in the collimated beam. This led to the first measurement of the focal spot, specifically the encircled energy. The size of the focal spot and the efficiency of the optics was then computed from this measurement. The test was performed at a wavelength of $390 \mathrm{~nm}$ from the LED with an angle of incidence of $3.5+/-0.01$ degrees and is illustrated in figure 2.

\subsection{Performances measured}

\subsubsection{Beam absolute measurement}

In order to compute an absolute calibration of the optics, the collimated beam was measured utilising the NIST photodiode. More than 150 measurement points were taken over the beam sec- 

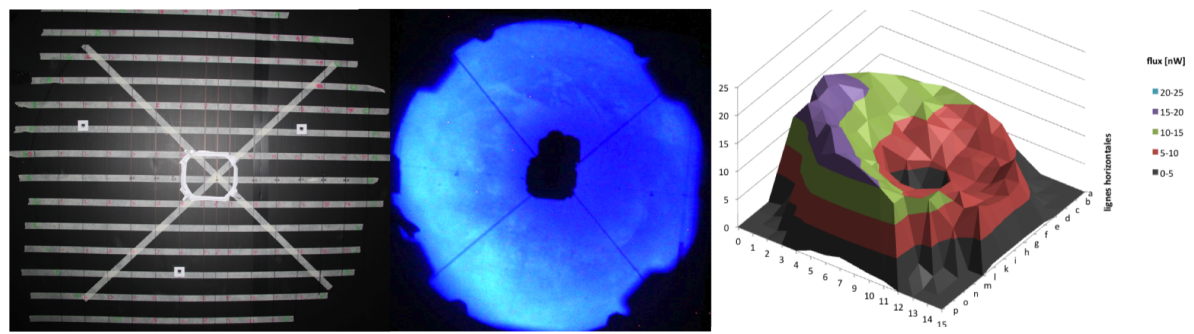

Figure 3: Measurements of the beam. From left to right: grid of NIST measurements, image of the UV beam and interpolated light power distribution of the beam. The grid is $1 \mathrm{~m} \mathrm{x} 1 \mathrm{~m}$ in size with $7 \mathrm{~cm}$ steps.
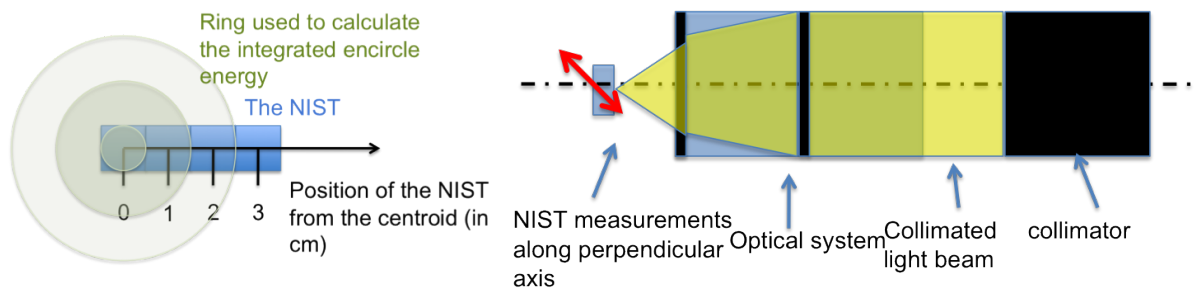

Figure 4: Left: Diagram describing the way the encircled energy is computed using the ring integration method. Right: Experimental setup. The collimated beam is sent through the optical system while the NIST measures light along an axis perpendicular to the optical axis.

tion on a regular grid described in figure 3. From this, the light power in the beam was determined to be $80.5 \mu W( \pm 4.4 \mu W \simeq 5 \%)$.

\subsubsection{Encircled energy}

The best way to measured the efficiency is to look at the encircled energy. The encircled energy is the amount of light contained in a certain circle centered on the centre of the optical spot. Knowing the light power collected on the aperture of the instrument, the encircled energy can be absolute-calibrated. Computing the encircled energy for different radii enables us to measure the shape of the spot and to calculate the efficiency and the throughput.

Due to the size of the focal spot, the NIST photodiode needed to be moved across the focal plane. After finding the best position for the focal surface along the optical axis ( $\simeq$ position measuring the maximum light flux), the focal plane was scanned with the NIST along an axis perpendicular to the optical axis. This measurement was used to compute the encircled energy at different distances from the centroid of the spot; i.e the light flux measured at the focal plane in a circle of a certain radius. For that, and knowing that the NIST active area as a surface of $1 \mathrm{~cm}^{2}$, the focal plane was split up into $1 \mathrm{~cm}$-thick rings. Measurements along the perpendicular axis enabled the light power within each ring to be approximated. The encircled energy was then computed by summing the energy contained within each ring at various distances from the centroid. This method is depicted by figure 4 .

The measured encircled energy is shown in figure 5. It increases quickly due to the central spot but never reaches a plateau within the focal surface of the PDM. This is due to the presence of an intense diffuse light that reaches the entire focal plane. We defined the efficiency of the optics as the absolute encircled flux, calibrated with the light power sent through the optics from the collimated 


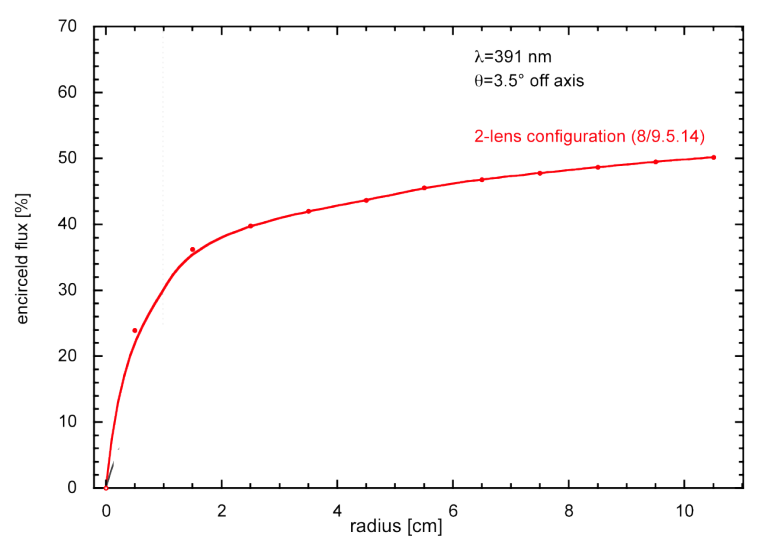

Figure 5: Encircled light energy measured with the collimated beam.
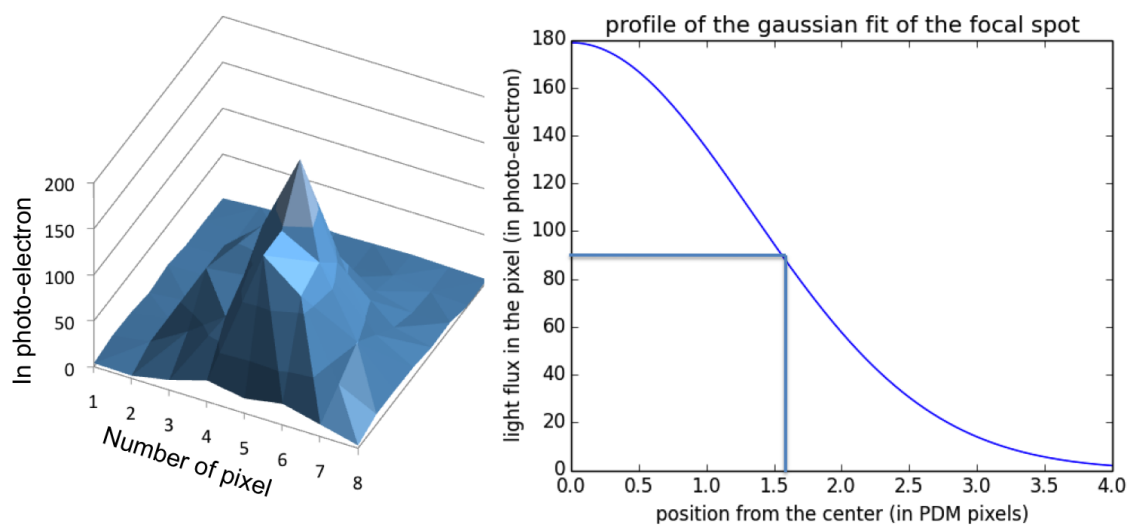

Figure 6: Left: Focal spot taken by the PDM. Right: 2-D gaussian fit of the focal spot. FWHM =9mm.

beam. Thus, the efficiency at 3.5 degrees and $390 \mathrm{~nm}$ is equal to $\mathbf{3 0 . 5 \%} \pm \mathbf{3}$ for the central area of $1 \mathrm{~cm}^{2}$ and $\mathbf{2 2 \%} \pm \mathbf{2 . 2}$ for an area $\mathbf{0} \mathbf{0 . 7 5 \mathbf { c m } ^ { 2 }}$, corresponding to the geometric area of $3 \times 3$ pixels of the real instrument detector, PDM (Photo Detector Module). Using the measurements made with the instrument detector PDM, we produced an image of the focal spot in order to fit a 2-dimensional Gaussian distribution. This led to a full width at half maximum of $\mathbf{9 m m} \pm \mathbf{0 . 2}$ as shown in figure 6. Therefore, according to this measurement, the efficiency of the optics to use in a study of an imaged object would be the one provided by a $0.75 \mathrm{~cm}^{2}$ spot: $\mathbf{2 2 \%} \pm \mathbf{2 . 2}$.

Measured over a large radius, the encircled energy can be used to compute the throughput, i.e. the total amount of light power received on the entire focal surface. The real detector, PDM, is a square of $165 \mathrm{~mm}$ by $165 \mathrm{~mm}$. Knowing the encircled flux is equal to $48 \%$ for a radius of $82.5 \mathrm{~mm}$ and $51 \%$ for a radius of $82.5 * \sqrt{2} \mathrm{~mm}$, we can compute an initial estimate value of the throughput of the optics. It is therefore circa $\mathbf{5 0 \%}$ for a PDM focal surface size.

\section{Long corridor test campaign}

\subsection{Description of the test setup}

For the long corridor test campaign, 'collimated light' was achieved by placing the light source 


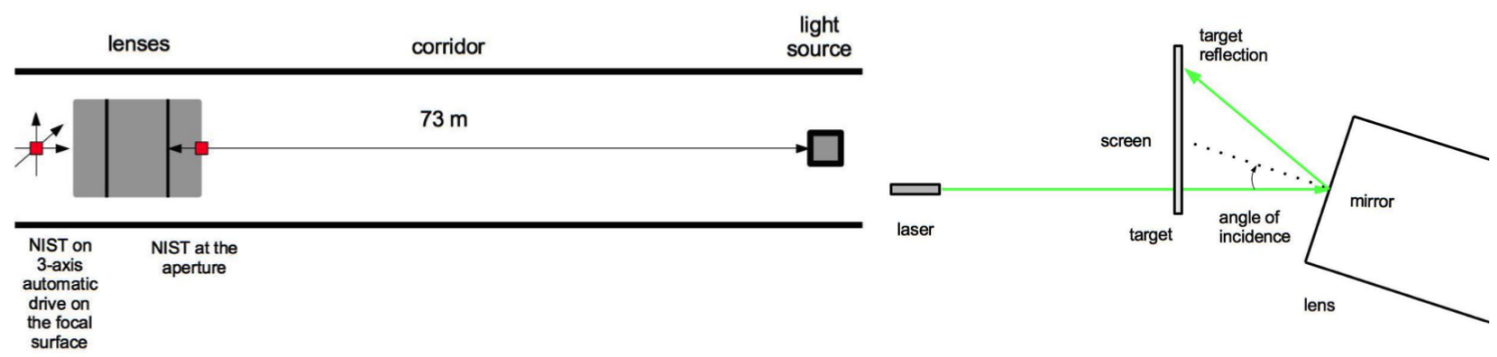

Figure 7: Description of the experimental setup. Left: A light source is placed far from the optical system $(73 \mathrm{~m})$ in order to produce a roughly parallel light beam at the instrument. The focal plane is measured by the NIST photodiode along the 3 axes. Right: The angle of incidence of the incoming beam is measured pointing a laser from the light source to the instrument. The position of the reflection of the laser on the first lens of the instrument is used to measure the angle of incidence.

at a position approaching infinity from the EUSO-Balloon optical system. This new setup enable us to measure at multiple wavelengths and angles of incidence more easily than with the collimator setup. A $100 \mathrm{~W}$ mercury lamp light source was positioned $73 \mathrm{~m}$ from the optical system as detailed in figure 7. Band-pass filters for $313 \mathrm{~nm}, 365 \mathrm{~nm}$ and $405 \mathrm{~nm}$ were positioned to transmit a specific UV wavelength to the optical system. Apertures and baffles were placed in front of the light source to eliminate any stray light emitted by the mercury lamp. The angle of incidence of the light source was determined by shining a laser from the mercury lamp to a small mirror at the centre of the L1 lens manifold. The angle was determined by observing the location of the laser's reflection, depicted in figure 7 .

To map the focal spot, the light flux received through lenses L1 and L3 was recorded by the NIST, which was fixed to a 3-dimensional translation stage behind L3. The micrometric translation stage can measure through a $300 \mathrm{~mm} \times 100 \mathrm{~mm} \times 100 \mathrm{~mm}$ range in the $\mathrm{x}, \mathrm{y}$ and $\mathrm{z}$ directions respectively. This configuration allows for the accurate mapping of the focal spot and the shape of focused beam. A second NIST was placed at the front of the EUSO-Balloon optical system to record the ambient light flux in order to monitor any fluctuations from the light source, and therein adjust the received light values accordingly. Using the same methodology as per the collimator test campaign, the encircled energy and optical efficiency were able to be determined.

\subsection{Performances measured}

Using the 3D scanning capability of the translation stage, the focal plane was mapped to determine the optimal distance from L3 to the NIST. The focal spot was thereby determined by observing the position at which the light flux peaks and is most intense. At this distance, the measurements of the focal plane provided the shape of the focal spot and the encircled energy. An image of the focal spot as shown by figure 8 can be obtained from those measurements.

The encircled energy was computed with a method similar to what was used for the collimator test setup. Here, the focal plane was split up into $1 \mathrm{~mm}$-thick rings, with measurements at the focal plane used to calculate the average light power contained in each ring. The encircled energy was then computed by summing the energy contained within each ring at different distances from the centroid. In order to get a more precise measurement, small increments were employed using the 


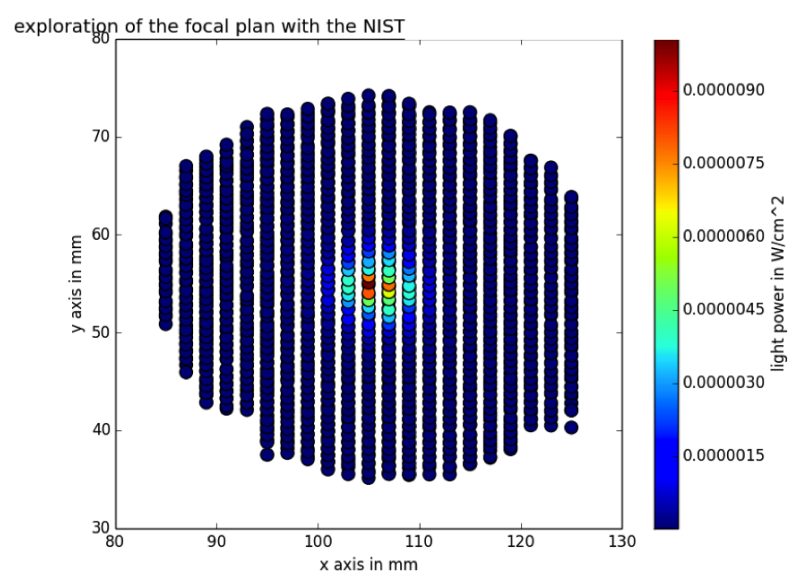

Figure 8: Image of the focal spot at $405 \mathrm{~nm}$ and 2.3 degrees at the best position along the optical axis. The horizontal and vertical axes origins are arbitrary.

translation stage and a $1.4 \mathrm{~mm}$-diameter diaphragm was placed on the NIST to reduce its receiving area.

So far, we have measured the encircled energy for three wavelengths, $313 \mathrm{~nm}, 365 \mathrm{~nm}$ and $\mathbf{4 0 5} \mathbf{n m}$, at one incident angle, 2.3 degrees $\pm \mathbf{0 . 1}$, as shown by figure 9 . Assuming that the focal spot size is the same as that measured by the first test campaign, i.e. $3 \times 3$ pixel size, we computed the efficiency at 2.3 degrees for each wavelength. These efficiencies are presented in table 1.

\begin{tabular}{l|c} 
Wavelength & Efficiency \\
\hline $313 \mathrm{~nm}$ & $16 \%$ \\
$365 \mathrm{~nm}$ & $21 \%$ \\
$405 \mathrm{~nm}$ & $26 \%$
\end{tabular}

Table 1: Efficiencies computed for a $0.75 \mathrm{~cm}^{2}$ spot size at an angle of incidence of 2.3 degrees

\section{Conclusion}

In this article, we have presented selected measurements of the characteristics of the optical system. The measurements were made during two test campaigns described in the article and characterisation is still ongoing. The first campaign used a collimator and a 390nm UV LED while the second campaign used a different setup with a polychromatic light source placed far away from the instrument. Different band-pass filters provided monochromatic light sources. An absolutecalibrated NIST photodiode was used to scan the entire the focal plane in order to measure the size of the spot and the encircled energy. From the encircled energy, the efficiency was computed.

The spot size was measured at one wavelength and one degree of incidence, and is geometrically equal to $3 \times 3$ pixels, approximately $9 \mathrm{~mm}$ width. This measurement could be used as an initial approximation of the focal spot size. The efficiency of the spot during the first campaign, measured 


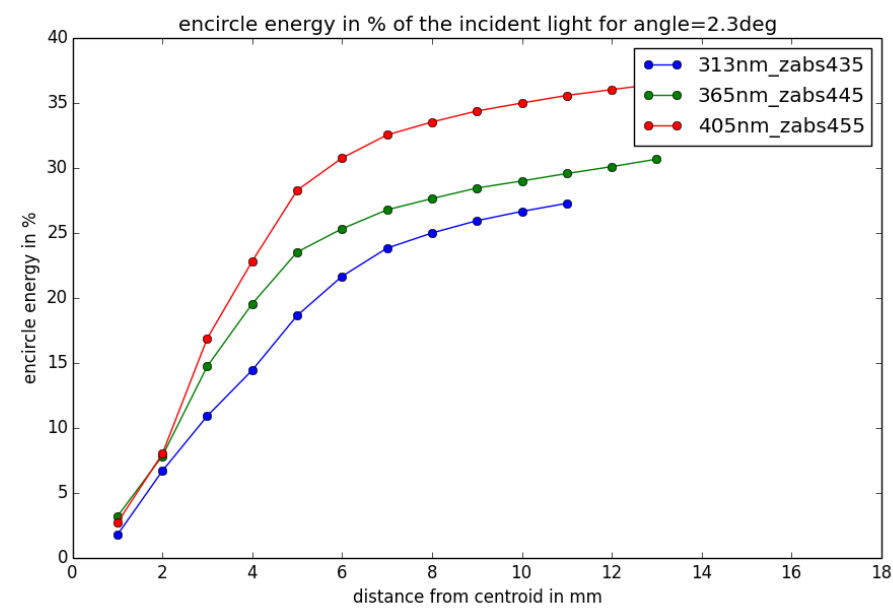

Figure 9: Encircled energy measured at 2.3 degree and at three wavelengths. For each wavelength, the measurement was taken at the best distance between L3 and the NIST, called "zabs". The encircled energy is normalised with the light power collected by the instrument.

at an angle of incidence of 3.5 degrees and 390nm wavelength, was $22 \%$. During the second campaign, the efficiency was measured at an angle of incidence of 2.3 degrees for three wavelengths, $313 \mathrm{~nm}, 365 \mathrm{~nm}$ and $405 \mathrm{~nm}$, yielding efficiencies of $16 \%, 21 \%$ and $26 \%$ respectively. These values, and any combination of them, should be used carefully as first measurements of the characteristics of the optics. Indeed, despite being confident in the results, the measurements are not complete and the characterisation needs to be completed at other wavelengths and angles. This efficiency must only be used for the study of imaged objects or point sources, such as the optical efficiency used to image air showers. It is not to be used in background light study. From the first campaign measurement, we have only an initial indication of the throughput of the optical system.This throughput should be used to study the background measured by EUSO-Balloon provided using future measurements at different wavelengths and different angles of incidence.

To summarise, in this article we have presented preliminary measurements of the characteristics of the EUSO-Balloon optical system for a number of angles of incidence and wavelengths: focal spot size, efficiency and throughput. Those numbers should be used as first approximations. Measurements are ongoing and full characteristics (at many wavelengths and angles of incidence) will be available soon, as well as combined computations.

\section{References}

[1] J.H. Adams Jr. et al. - JEM-EUSO Collaboration, Astroparticle Physics 44 (2013) 76

[2] P. von Ballmoos et al. 34nd ICRC, Rio de Janeiro, 2015

[3] Y. Takizawa et al. 33nd ICRC, Rio de Janeiro, 2013 